\title{
SUPPRESSING THE SURFACE FIELD DURING TRANSCRANIAL MAGNETIC STIMULATION
}

By:

K.R. Davey

M. Riehl

IEEE Transactions on Biomedical Engineering Mark Riehl: Neuronetics, Inc.

PR - 375

Center for Electromechanics

The University of Texas at Austin

PRC, Mail Code R7000

Austin, TX 78712

(512) 471-4496

March 18, 2004 


\title{
Suppressing the Surface Field during Transcranial Magnetic Stimulation
}

\author{
Kent R. Davey, IEEE Fellow and Mark Riehl
}

\begin{abstract}
Transcranial Magnetic Stimulation (TMS) is used commonly as both a diagnostic tool and as an alternative to electric shock therapy for the treatment of clinical depression. Among the clinical issues encountered in its use is the mitigation of accompanying pain. The objective becomes one of minimizing the induced surface field while still achieving the target field objective. Three techniques discussed for realizing this end are (1) placing a conducting shield over a portion of the central target region, (2) using supplementary coils of opposite polarity in tandem with the primary field, and (3) opening the core angle to distribute the field. Option (3) shows the greatest promise for reducing the ratio of the maximum surface field to induced target field
\end{abstract}

Index Terms - electric field, iron core, magnetic, stimulation, suppression

\section{INTRODUCTION}

$\mathrm{T}$ RANSCRANIAL magnetic stimulation is a noninvasive technique for stimulating cortical neurons by a rapidly changing magnetic field [1]. It has been used in neurology as a diagnostic tool and in functional brain mapping [2] [3]. In recent years, TMS has proved quite useful as an alternative to electroshock therapy for the treatment of depression and Parkinson's disease [4] [5]. Unfortunately, one of the side effects is pain. In a special issue on TMS, Wasserman states "rTMS (repetitive TMS) can cause significant local discomfort due to the direct activation of nerves and muscles in the scalp." [6] The pain experienced varies among patients, and is quite dependent on the excitation level and position of the stimulator. The surface field near the scalp appears to be the primary source of the problem, inducing pain by surface depolarization of trigeminal nerve branches in the scalp due to the higher field concentration in this region. The induced field on the scalp is stronger than in the cortex. Iron core magnetic stimulators aggravate this problem because of the concentration of the flux. This paper examines some ways in which the surface field on the scalp can be minimized while still achieving the deeper stimulation within the cortex. Among the possible solutions examined are the following:

1) Use a conducting surface shield to suppress the induced field beneath the shield.

2) Use a smaller supplementary coil to oppose the primary field near the center of the stimulation core.

3) Increase the stimulation core opening angle.

All three options require additional stimulation energy.

Fig. 1's inset shows a picture of $1 / 4$ of the principle core analyzed in this document. The core is a tape wound magnetizable core, typically comprised of $3 \%$ grain oriented steel. Tape wound cores substantially reduce the required system size and energy requirements [7] [8], although their construction is more difficult [9]. Most of the principles considered apply to air core stimulators, but magnetizable cores will remain the focus in this document because of their growing use in industry. Neotonus, Inc. is using magnetizable cores for the treatment of incontinence; they have performed over 2,000,000 treatments in over 300 clinics in 35 countries. Another company, Neuronetics, Inc., is using these cores for the treatment of depression. Seratonin release is related to action potential termination at a synaptic junction.

\section{IMPORTANCE OF THE PROBLEM}

The use of strong electric fields in the treatment of many neurological disorders is well established. Both in the treatment of incontinence and clinical depression, the electric field should be sufficiently strong to initiate an action potential. A changing magnetic field induces an electric field within a conducting medium. Inducing the electric field is valuable for many reasons. It allows treatment through patient clothing, and it is a convenient means of getting past the skull for transcranial stimulation.
Manuscript received March 18, 2004. This work was supported in part by Neuronetics, Inc.

K. R. Davey is with the Center for Electromechanics, University of Texas, 10100 Burnet Rd, PRC EME 133, Austin, TX 78758, USA. (512-232-1603, fax 512-471-0781, k.davey@mail.utexas.edu).

Mark Riehl is with Neuronetics, Inc., One Great Valley Parkway - Suite 2, Malvern, PA 19355 (mriehl@neuronetics.com). 

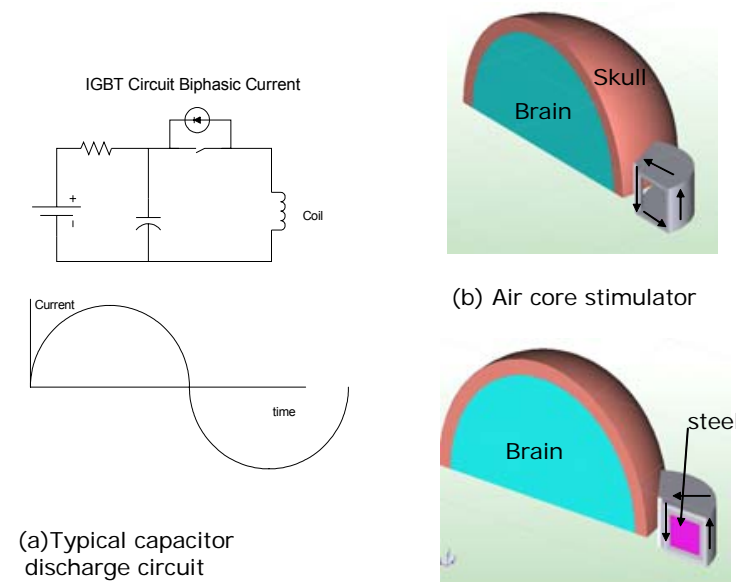

(b) Air core stimulator

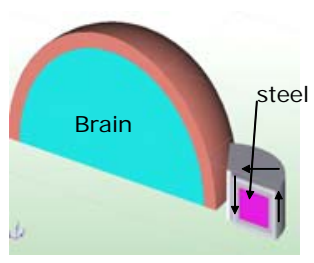

(c) Iron core stimulator

Fig. 1 (a) Typical magnetic stimulation circuit, (b) $1 / 2$ of an air core stimulator for TMS, and (c) an iron core stimulator showing a winding wrapped around a tape wound laminated core..

Nearly all magnetic stimulators work by discharging a capacitor into an inductor as suggested in Fig. 1. The simplest magnetic stimulator is a coil of wire around a magnetic core, such as that suggested in inset $\mathrm{c}$. Iron core stimulators require a specially constructed tape wound core to suppress eddy currents. These tape wound cores require about $1 / 4$ of the energy of air core stimulators to realize the same induced electric field.

The importance of this problem surfaces because the induced electric fields are indeed proving effective, but their use is usually accompanied by pain, especially with transcranial magnetic stimulation. Both the magnetic field and the induced electric field fall off exponentially with distance from the core in the near field region. Neurons in the soft scalp are subjected to the strongest field. Another area which registers problems is within neurons passing through an opening in bone. This paper focuses on the first problem. It is an oxymoron to think about a no surface field magnetic stimulation. There are, however, means by which the ratio of target depth electric field to surface field can be reduced considerably. This paper analyzes methods for achieving this objective without introducing additional sources.

One option for suppressing the surface field is to superimpose a secondary electric field with electrodes. The idea is shown in Fig. 2. Surface electric fields are placed normal to the induced surface field. Every other electrode must be excited with independent potential sources and chosen to inject a current opposite to the induced current. The skull ensures that the injected current will not penetrate into the white and grey matter. This option will not be discussed because of the difficulty of implementation.

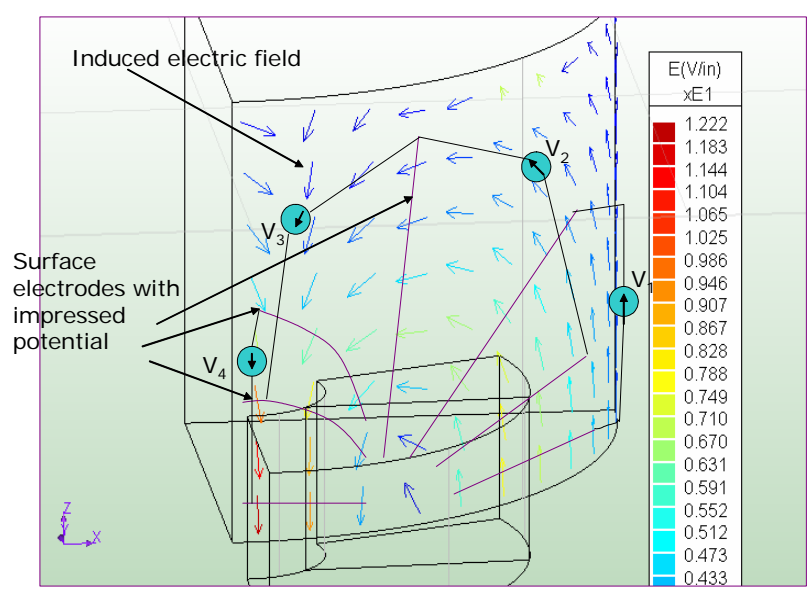

Fig. 2 Surface electrodes can be placed on the scalp on equipotential lines. Because of the demand placed on the placement and contact of the electrodes, as well as the excitation, this approach is not recommended.

The electric field results displayed in this paper are computed using a boundary element solver [10][11]. For simplicity and speed, the results are displayed and computed in two dimensions, through a midplane cut through the stimulation core.

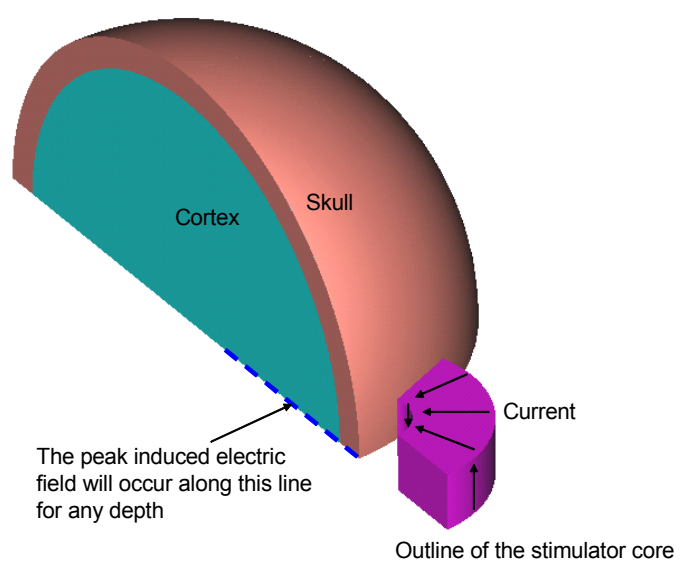

Fig. 3 Stimulator core next to the brain in quarter plane perspective.

\section{SURFACE SHIELD}

A surface conductor has the ability to suppress the local electric field, and in fact drive it to zero under the conductor if it is in electrical contact with the scalp. The integral form of Faraday's law,

$$
\oint \vec{E} \cdot d \vec{l}=-\frac{d}{d t} \int \vec{B} \cdot \hat{n} d S,
$$

makes it clear that this technique will both move and increase the magnitude of the peak field. A better approach is to insulate the conductor from the surface. 


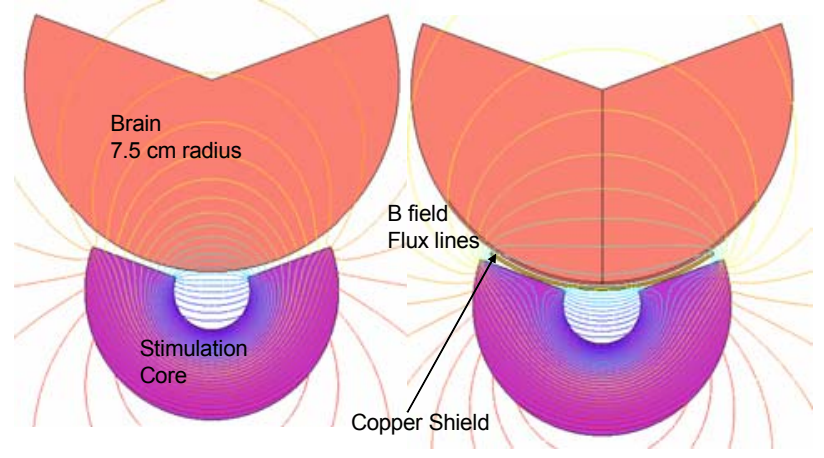

(a) TMS excitation topology

Fig. 4 Transcrannial magnetic stimulation B field plot without (a) and with (b) a passive copper shield.

Consider the TMS setup shown in Fig. 4. The stimulation core is considered to be a tape wound $3 \%$ silicon grain oriented core excited at 5,295 AT, $5.208 \mathrm{kHz}$ for one pulse. The brain is modeled as a homogeneous medium of conductivity $0.48 \mathrm{~S} / \mathrm{m}$, the conductivity of white matter. The steep fall off of the field from the core plotted in inset (a) is characteristic of the ferromagnetic stimulator core. If the core has an outer radius of $5.08 \mathrm{~cm}$, and an inner radius of $1.53 \mathrm{~cm}$, it induces an electric field of $1 \mathrm{~V} / \mathrm{cm}$ at a depth of $3 \mathrm{~cm}$. The field value of $1 \mathrm{~V} / \mathrm{cm}$ is considered to be close to threshold for cortical axon stimulation.

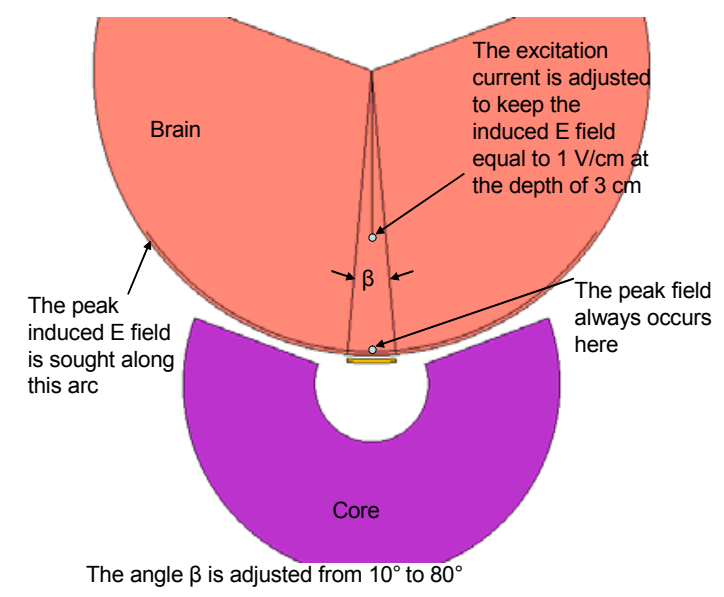

Fig. 5 Changing the angle of the shield conductor reduces the maximum induced field on the scalp.

Consider varying the opening angle of the shield conductor as suggested in Fig. 5. As the angle gets larger, the shield reduces the induced electric field. As the shield angle increases, increase the current exciting the core so that the induced electric field at the target depth of $3 \mathrm{~cm}$ remains fixed at $1 \mathrm{~V} / \mathrm{cm}$. At each test, compute the maximum induced electric field along the scalp $1 \mathrm{~mm}$ in from the surface. For this topology, the peak E field $1 \mathrm{~mm}$ below the surface always

occurs on the centerline of the core. This result changes a bit in $3 \mathrm{D}$ analysis.

Fig. 6 quantifies the reduction realized as the angle of the shield conductor is increased. Inset (a) shows the maximum induced surface E field (1 mm depth) as a function of shield angle, while maintaining the target E field at $100 \mathrm{~V} / \mathrm{m}$. When $\beta$ is increased to $80^{\circ}$, the induced $E$ field on the surface is reduced by about $31 \%$. The excitation current in the core, coincidentally, increases by $40 \%$ to maintain the same target excitation field. Fig. 4(b) clearly shows that the effect of the shield conductor is to open up the field, increasing the effective opening of the core.
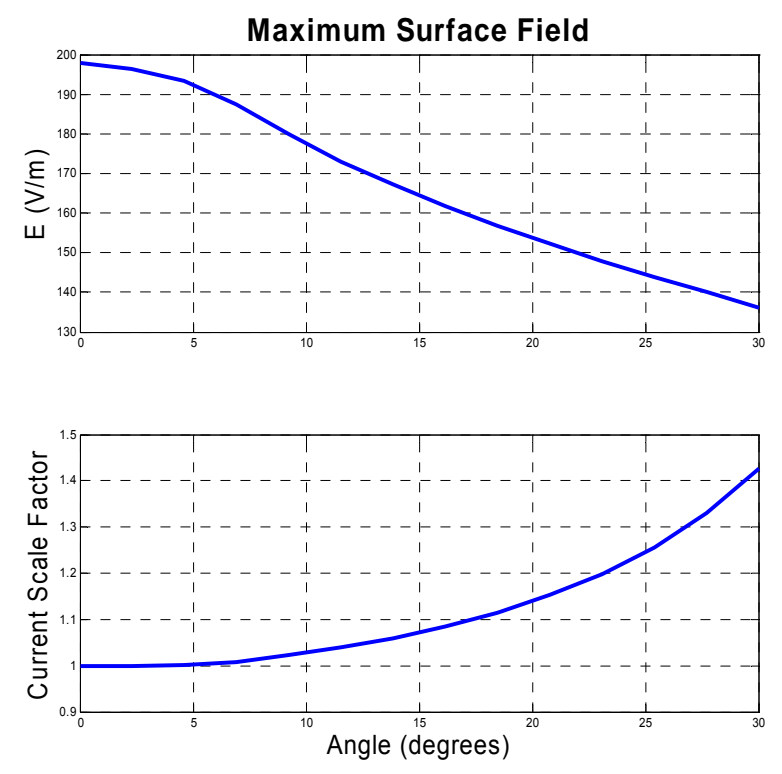

Fig. 6 Reduction in surface E field with increased shield conductor opening angle.

There still remains the question of whether this solution is efficacious. The criteria for a neuron to fire is that the intercellular potential rise by about 30 to $40 \mathrm{mV}$. During TMS, the intercellular potential is being driven up by the influx of positive ions. When the electric field is collinear with the neuron, and the neuron is relatively long, the gradient of the electric field will dictate the condition necessary for an action potential. However, at axon bends, branch points, and terminations, the E field, not its gradient, becomes the dominant factor. Axons deep within the brain have a predominant radial orientation. Consider how the $\mathrm{E}$ field gradient changes along the vertical bisection line shown in Fig. 4(b). The TMS core stimulator without shield induces an E field with a pronounced gradient, the field falling off roughly as $\exp (-\mathrm{x} / \lambda)$, where $\lambda$ is the opening of the $\mathrm{C}$ core. Fig. 7 shows a comparison of the shielded and unshielded electric field gradient. It is clear that the copper shield accomplishes a minimization of the surface electric field at the expense of the $\mathrm{E}$ field gradient. The topology of neurons within the first $2-3 \mathrm{~cm}$ below the scalp is complicated, but is generally characterized by axons with bends so that at least a portion of the axon is tangential to the skull. Because the cortex is dominated by synapses, focusing on the E field, 
rather than the $\mathrm{E}$ field gradient, is warranted for this document.

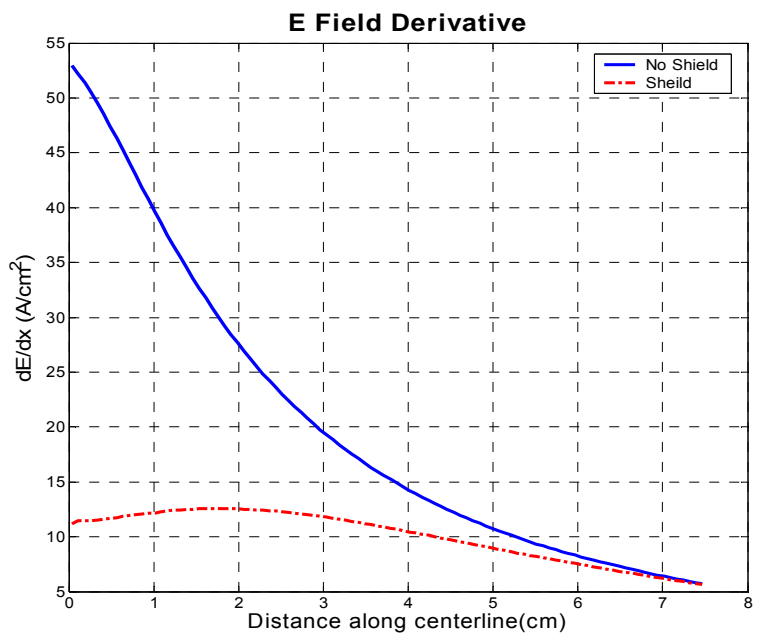

Fig. $7 \mathrm{E}$ field gradient along a radial line centered at the core.

\section{A. Reverse Excited Secondary Coils}

Fig. 8 shows how a secondary coil can be inserted within the primary coil. If the secondary coil has a reverse excitation from the primary winding, it will lower the surface field. Because the secondary coil is an air core winding, and the winding spread is small, the field penetration into the brain is reduced. Fig. 9 shows that the suppression of the $\mathrm{E}$ field occurs, but at a price. The E field is plotted along the scalp at a depth of $1 \mathrm{~mm}$. The primary core current is adjusted with and without the exciter coil to deliver $1 \mathrm{~V} / \mathrm{cm}$ at a depth of 3 $\mathrm{cm}$ within the brain. This $9.1 \%$ improvement in surface field is obtained with a secondary exciter current $41 \%$ of the primary current. The improvement can be increased to $12.6 \%$ by distributing the return current.

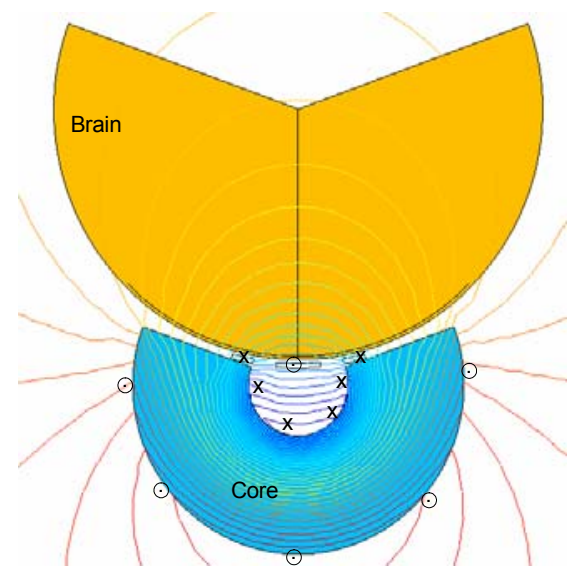

Fig. 8 Reverse excited secondary coil within to suppress the surface E field.

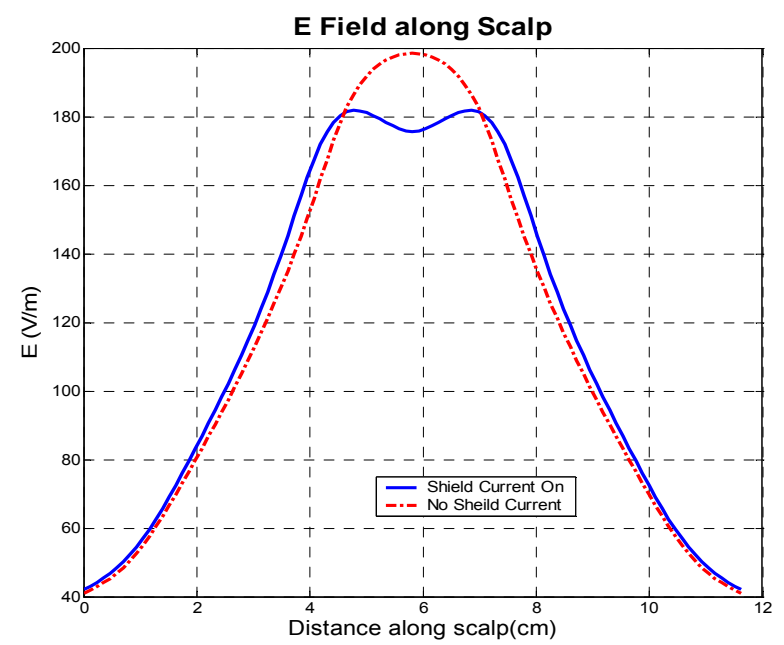

Fig. 9 The induced E field along the scalp is smaller when a secondary exciter coil is used.

\section{B. Stretched Core}

Shown in Fig. 10 is a magnetic core stimulator with the angle opened considerably from Fig. 3. Keep the 3\% grain oriented steel within the core. Inject the opposite current over a region spanning an angle $\beta$. The electric field is suppressed along the vertical midline. Fig. 11 shows the electric field along the scalp induced by this configuration. The primary core excitation is 3,285 A (per side) with 500 A being sent through the reverse excited coil in the center. Both this current and the angle $\beta=21^{\circ}$ are determined through optimization using variable metrics. The minimum induced $\mathrm{E}$ field that satisfies the target value of $1 \mathrm{~V} / \mathrm{cm}, 3 \mathrm{~cm}$ down, is $150 \mathrm{~V} / \mathrm{cm}$. The induced $\mathrm{E}$ field is computed along the complete arc annotated in Fig. 10.

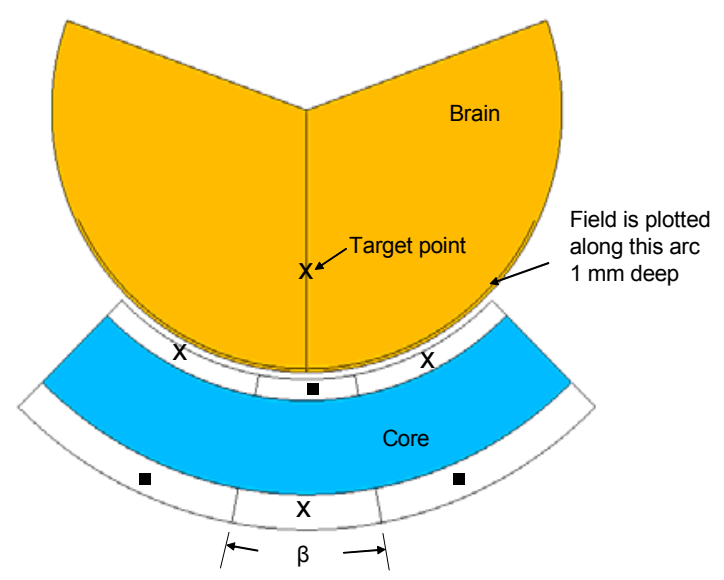

Fig. 10 Opening the angle of the core yields deeper field penetration. 


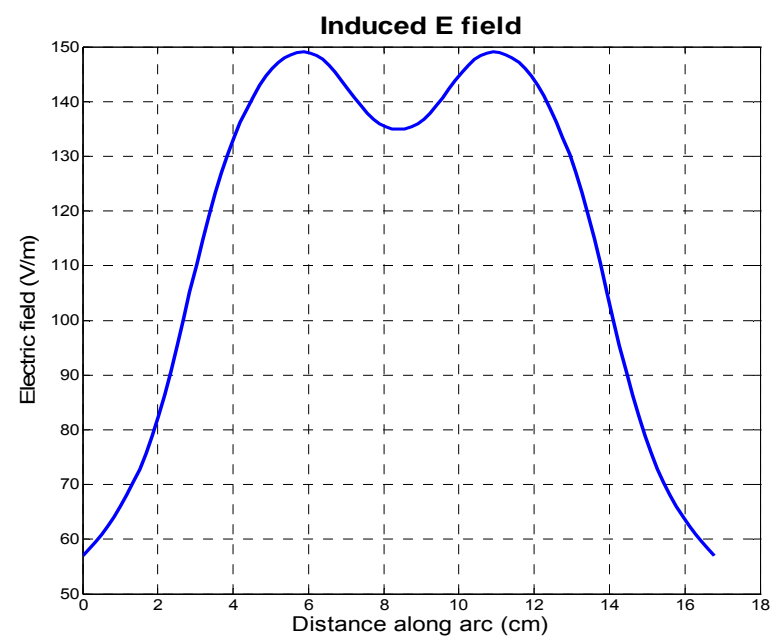

Fig. 11 Electric field along the scalp from the configuration in Fig. 10.

\section{Stretched Core, No reversed excitation winding}

Fig. 12 shows a simpler core without reversed excitation. Consider the exercise of opening the core angle from $90^{\circ}$ to $140^{\circ}$. At each opening, compute the correct excitation under saturation so that the induced E field $3 \mathrm{~cm}$ down remains at 1 V/cm. Fig. 13 shows the peak surface field and required core excitation as a function of core angle. The peak field has been reduced to $38 \%$ of its initial value in Fig. 4(a).

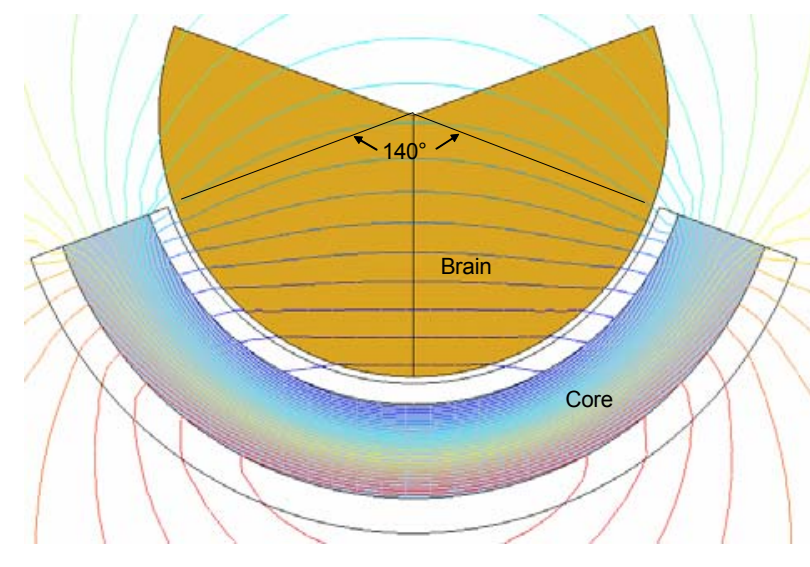

Fig. 12 Core opened to $140^{\circ}, 3790 \mathrm{AT}$ excitation at $5280 \mathrm{~Hz}$.
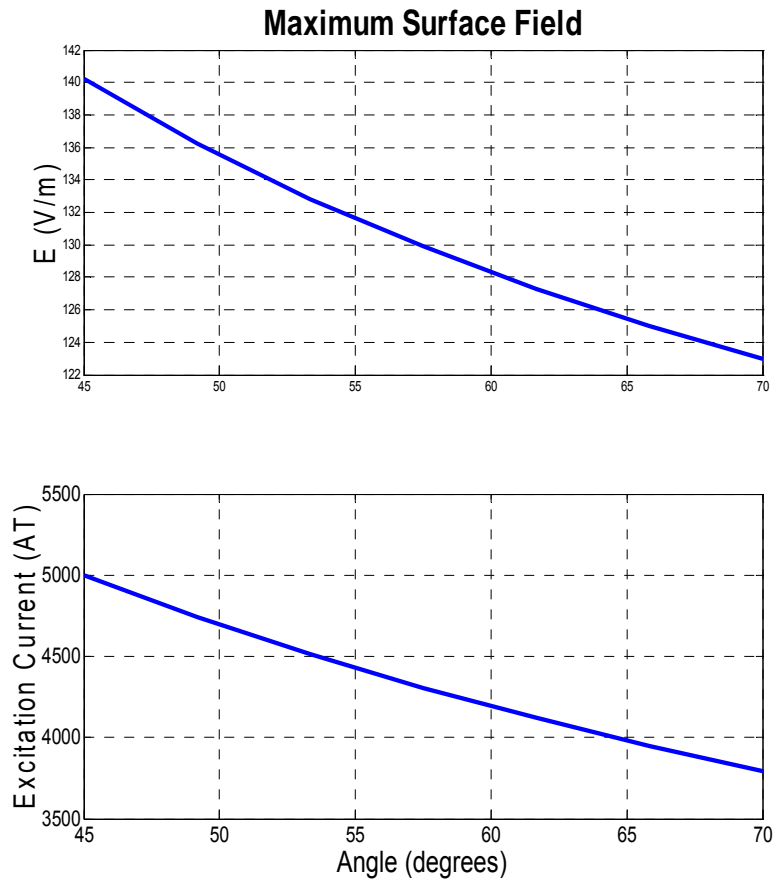

Fig. 13 Peak surface field and amp-turn excitation with increasing core angle.

\section{A Comparison of the Three Methods}

Three methods have been suggested for mitigating the surface field, using a passive conducting shield, actively exciting a smaller coil with opposite field polarity, and opening up the excitation core angle. Of the three methods, the third is favored. The reverse field excitation has the drawback that it requires either another power supply or additional load to the existing supply. This method has a safety issue; if the reverse field excitation fails or suffers a phase lag, the patient will suddenly experience much pain. The passive shield is rated second because there is little probability that it will fail. However it imposes an additional load on the stimulator supply. The more serious difficulty is the heat dissipation within the shield. Continuous excitation will register a possibly dangerous rise of the shield temperature. The open angle core poses the least additional burden on the stimulator power supply, and it does not suffer the safety problems of the reverse excitation or thermal shield.

\section{CONCLUSIONS}

Suppressing the surface field during transcranial magnetic stimulation has been accomplished to a limited extent through the use of passive conductor shields, supplementary coils of opposite polarity excitation, and changing the core angle. The latter method shows the greatest success. All require clinical testing. Additional methods exist, such as electrically injecting current through electrodes during the magnetic field transient, and may warrant attention for future work. Preliminary tests show that these techniques offer little reduction in the maximum field, but are useful in moving that maximum to another location. Surface electrodes on the scalp are expected 
to be problematic because of implementation, surface contact, and additional excitation source requirements.

The root of the problem is pain experienced clinically. The techniques examined in this paper are under investigation. None have gone through clinical testing, although the surface shield has been tested by Neuronetics, Inc. internally. It does reduce surface pain. Procurement of the open angle core and subsequent tests are yet to follow.

\section{REFERENCES}

[1] A. T. Barker, R. Jalinous, and I. L. Freeston, "Non-invasive magnetic stimulation of human motor cortex," Lancet, vol. 1, May 1985, pp. 11061107.

[2] M. Ogiue-Ikeda, S. Kuwato, and S. Ueno, "The effect of transcranial magnetic stimulation on long term potential in rat hippocampus," IEEE Trans. Magn., vol. 39, no. 5, Sept. 2003, pp. 3390-3392.

[3] J. R. Gates, "Transcranial magnetic stimulation," Neuromag. Clin. North Amer., vol. 5, Nov. 1995, pp. 711-720.

[4] A. Pascual-Leone, B. Rubio, F. Pallardo, and M. D. Catala, "Rapid-rate transcranial magnetic stimulation of left dorsolateral prefrontal cortex in drugresistant depression," Lancet, vol. 348, July 1996, pp. 233-237.

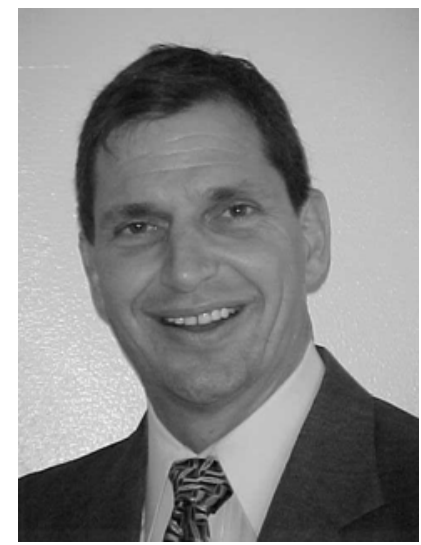

Kent Davey became a Member of IEEE in 1980, a Senior Member in 1986, and a Fellow in 2004. He was born in New Orleans, Louisiana, 1952. He received his BS in EE from Tulane in 1974, his MS in Power Engineering from Carnegie Mellon in 1976, and his MS in physics from the University of Pittsburgh in 1976. He received his $\mathrm{PhD}$ in EE from the Massachusetts Institute of Technology in 1980. He completed a Fulbright in Finland on atmospheric physics in 1981 .

From 1974 to 1976 he worked at Westinghouse as a large turbine generator designer. From 1979 to 1980 he served as an assistant professor at Texas A\&M University. From 1980 to 1994 he served as a tenured associate professor at the Georgia Institute of Technology. From 1994 to 2002, he served as technical director of American Maglev Technology in Edgewater, Florida. He presently serves as a research scientist at the Center for Electromechanics at the University of Texas in Austin, Texas. Dr. Davey has research activity in electromechanical machine design, pulsed electromechanical devices, HTS Trapped Field magnets, electroporation, and magnetic stimulation of biological tissue.

Dr. Davey is a Fellow with IEEE and presently serves as editor of IEEE Transactions on Magnetics. He is actively involved in the review of conference and journal papers for various IEEE activities.
[5] J. Mally and T. W. Stone, "Improvement in Parkinsonian symptoms after repetitive transcranial magnetic stimulation," J. Neurol. Sci., vol. 62, Jan. 1999, pp. 179-184.

[6] E. Wasserman, "Repetitive transcrannial magnetic stimulation: an introduction and overview," CNS Spectrums, vol. 2, no. 1, Jan. 1997, pp. 2125.

[7] K. R. Davey, C. H. Cheng, and C. M. Epstein, "An alloy - CORE electromagnet for transcranial brain stimulation," Journal on Clinical Neurophysiology, vol. 6, no. 4, 1989, pg. 365.

[8] C. M. Epstein and K. R. Davey, "Iron-core coils for transcranial magnetic stimulation," Journal of Clinical Neurophysiology, vol. 19, no. 4, Dec. 2002, pp. 376-381.

[9] K. Davey and C. M. Epstein, "Magnetic stimulation coil and circuit design," IEEE Transactions on Biomedical Engineering, vol. 47, no. 11, Nov. 2000, pp. 1493-1499.

[10] D. Zheng, K. R. Davey, R. Zowarka, and S. Pratap, "Pushing the limits of 2-D boundary element eddy current codes - connectivity," International Journal of Numerical Modeling: Electronic Networks, Devices and Fields, vol. 9, 1996, pp. 115-124.

[11] Y. B. Yildir, K. M. Prasad, and D. Zheng, "Computer aided design in electromagnetic boundary element method and applications," Control and Dynamic Systems, vol. 59, 1993, pp. 167-223.

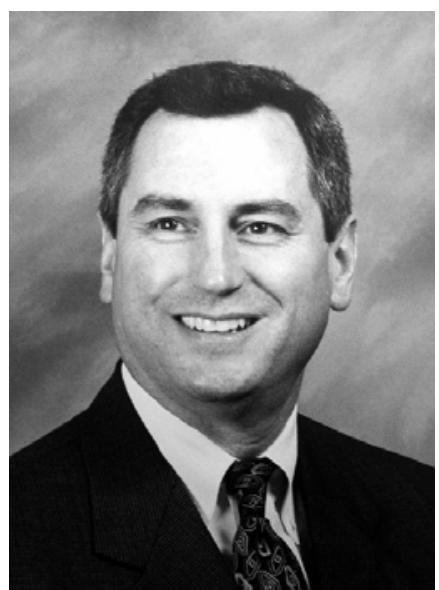

Mark E. Riehl became a Member (M) of IEEE in 1974 and again in 2003. $\mathrm{He}$ holds BS and MS degrees in electrical engineering from Purdue University, West Lafayette, IN (1972 and 1974 respectively) and completed additional graduate work in biomedical engineering at Marquette University, Milwaukee, WI (1977-1981).

He began his career at General Electric Space Division in 1974 working on satellite and flight simulator development and moved to GE Medical Systems in 1977. He was a Design Engineer in X-ray Products until 1981 when he began work on GE's first magnetic resonance imaging products. He was Manager of MR Systems Development Engineering and MR Engineering Programs from 1983 to 1994. From 1994 to 1998 he served as Vice President of Engineering at Dräger Medical, developing anesthesia and patient monitoring equipment. From 1998 to 2000 he served as Vice President of Instrument Engineering at Cytometrics and as Director of Product Development at Neuron Therapeutics. He has served as Vice President, Product Development and Operations for Neuronetics, Inc, Malvern, PA since its incorporation in April 2003, and presently holds that position. Areas of technical interest include biological effects of electromagnetic fields, diagnostic image processing, system architecture, medical device development and neurostimulation. 